\title{
Quantitative proteomic analysis of the central amygdala in neuropathic pain model rats
}

Kersti Karu¹, Richard S. Swanwick ${ }^{1}$, Amparo Novejarque-Gadea ${ }^{2}$, Ana Antunes-Martins ${ }^{3}$, Benjamin Thomas ${ }^{6}$, Eiji Yoshimi $^{1}$, William Foster ${ }^{1}$, Min Fang ${ }^{4}$, Stephen B. McMahon ${ }^{3}$, David L.H. Bennett ${ }^{5}$, Andrew S.C. Rice ${ }^{2}$, and Kenji Okuse ${ }^{* 1}$

${ }^{1}$ Department of Life Sciences, Faculty of Natural Sciences, Imperial College London, London SW7 2AZ, U.K. 2Department of Surgery and Cancer, Faculty of Medicine, Imperial College London, London SW10 9NH, U.K. ${ }^{3}$ The Wolfson Centre for Age-Related Diseases, King's College London, Guy's Campus, London SE1 1UL, U.K. ${ }^{4}$ Public Health England, London SE1 8UG, U.K.

${ }^{5}$ Nuffield Department of Clinical Neurosciences, University of Oxford, Oxford OX3 9DU, U.K.

${ }^{6}$ Central Proteomics Facility, Sir William Dunn School of Pathology, University of Oxford, Oxford, UK

\section{list of supplementary components}

Supplementary Figure S1: Nissl staining image showing rat amygdala

Supplementary Figure S2: 2D-PAGE images show protein changes in CeA of naïve and SNT models Supplementary Figure S3: 2D-gel of the left-side CeA

Supplementary Figfure S4: The entire gel image for the Western blot (GAPDH) 


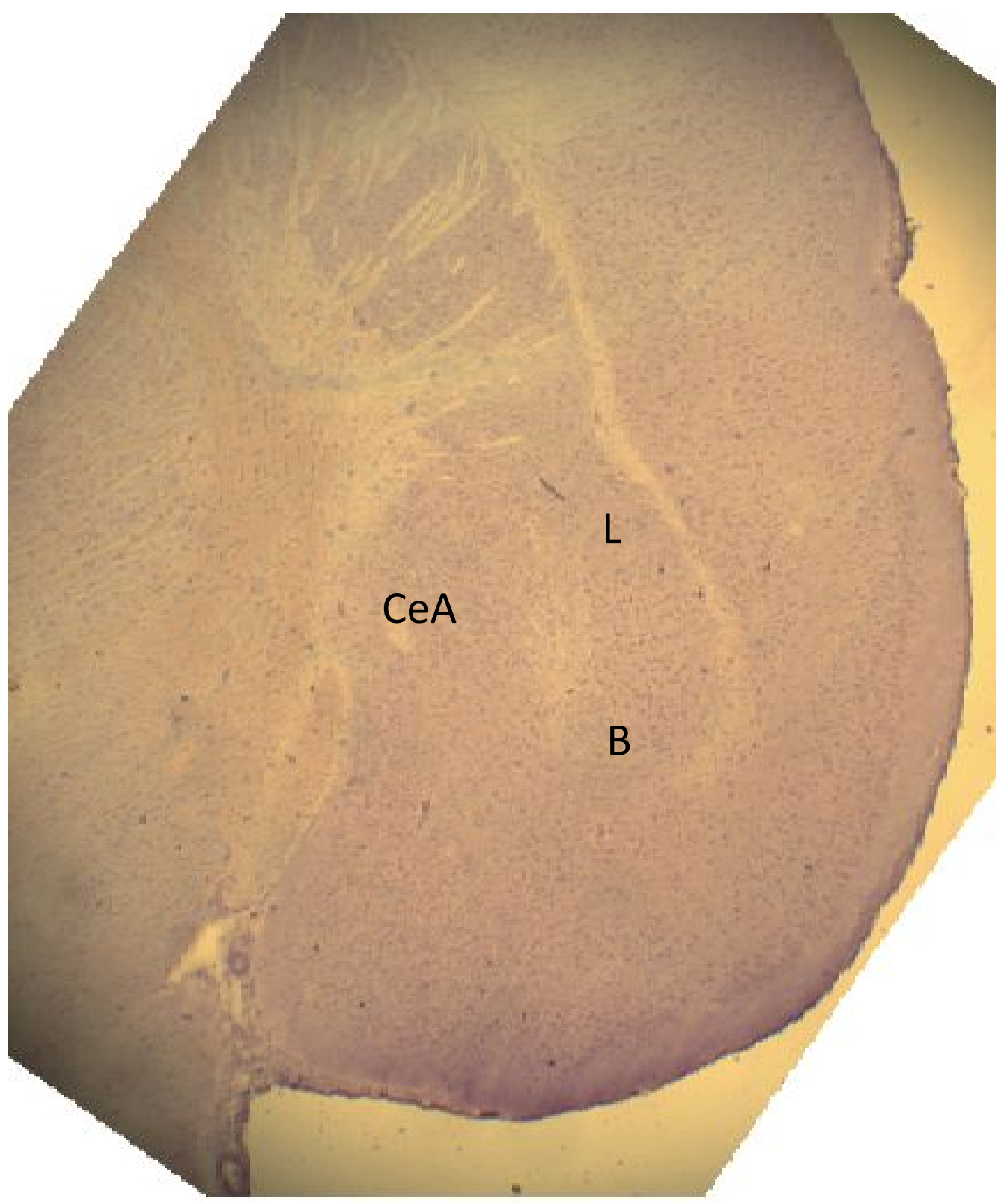

Figure S1. Nissl staining image showing rat amygdala (Stereotaxic location at -1.8 relative to bregma) in sham animal. Boundaries of the regions counted, the central nuclei $(\mathrm{CeA})$, the basl nuclei $(\mathrm{B})$, and the laterl nuclei $(\mathrm{L})$. Scale bar, 350 $\mu \mathrm{m}$. 
(A)

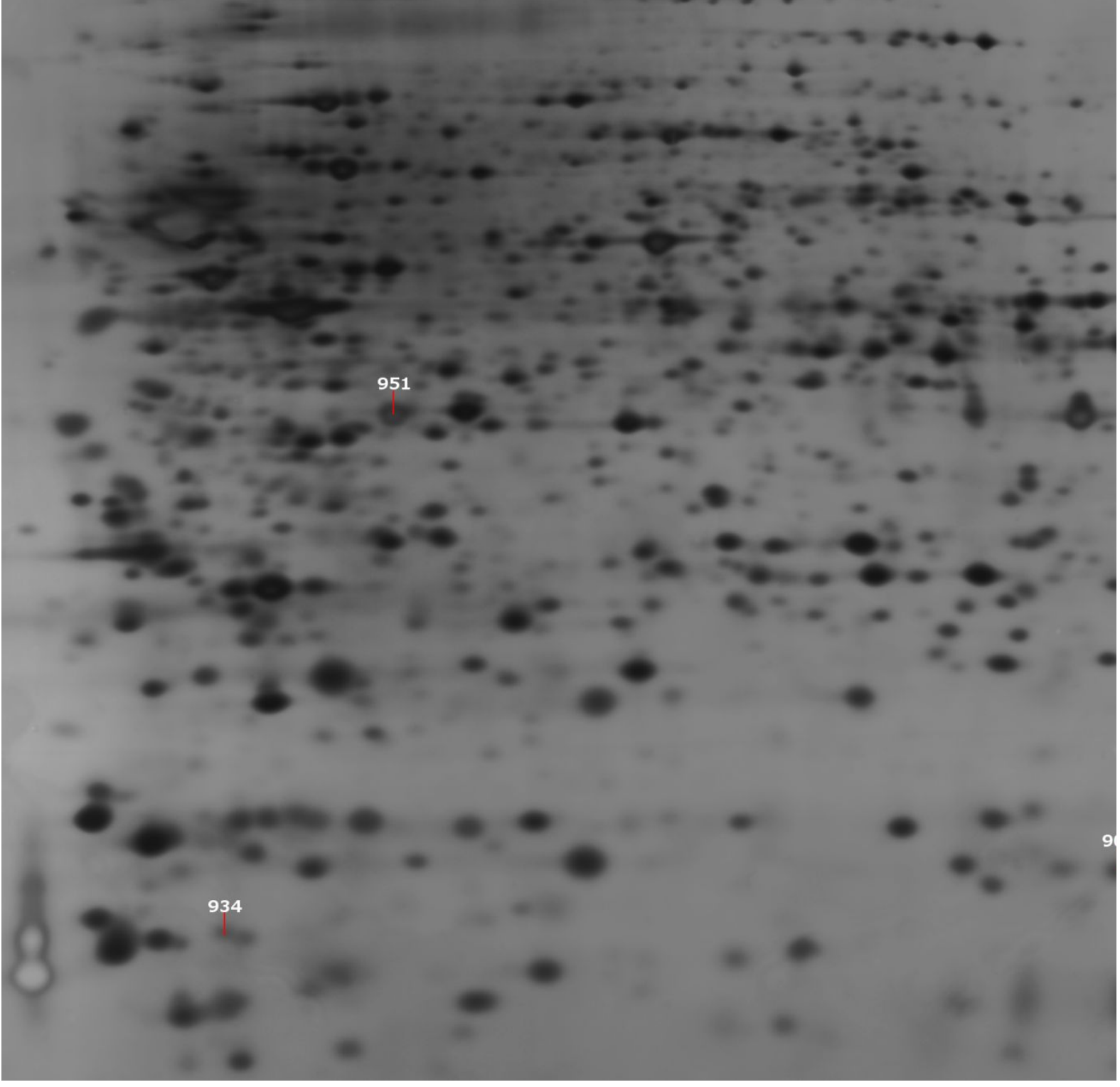


(B)

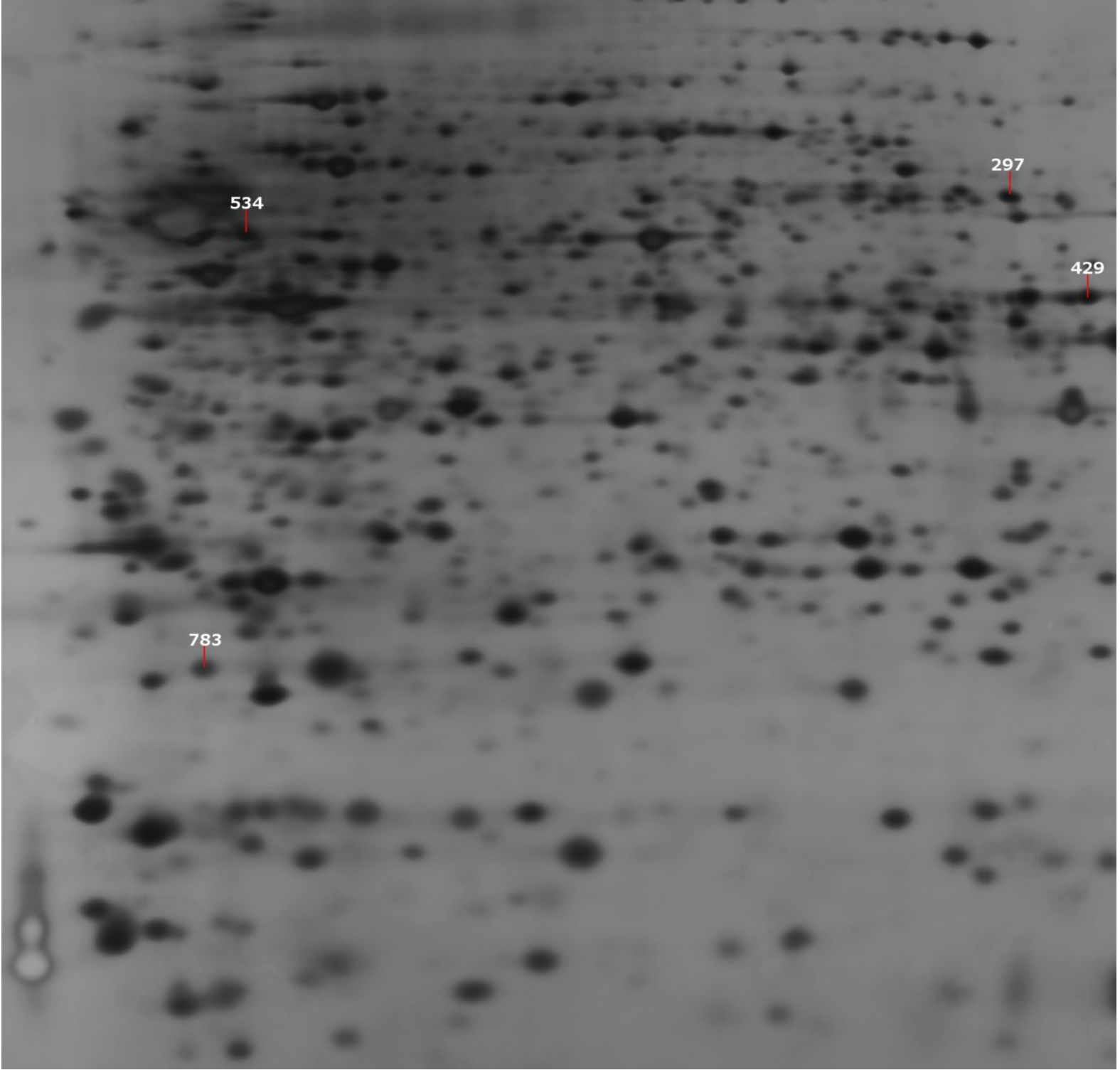


(C)

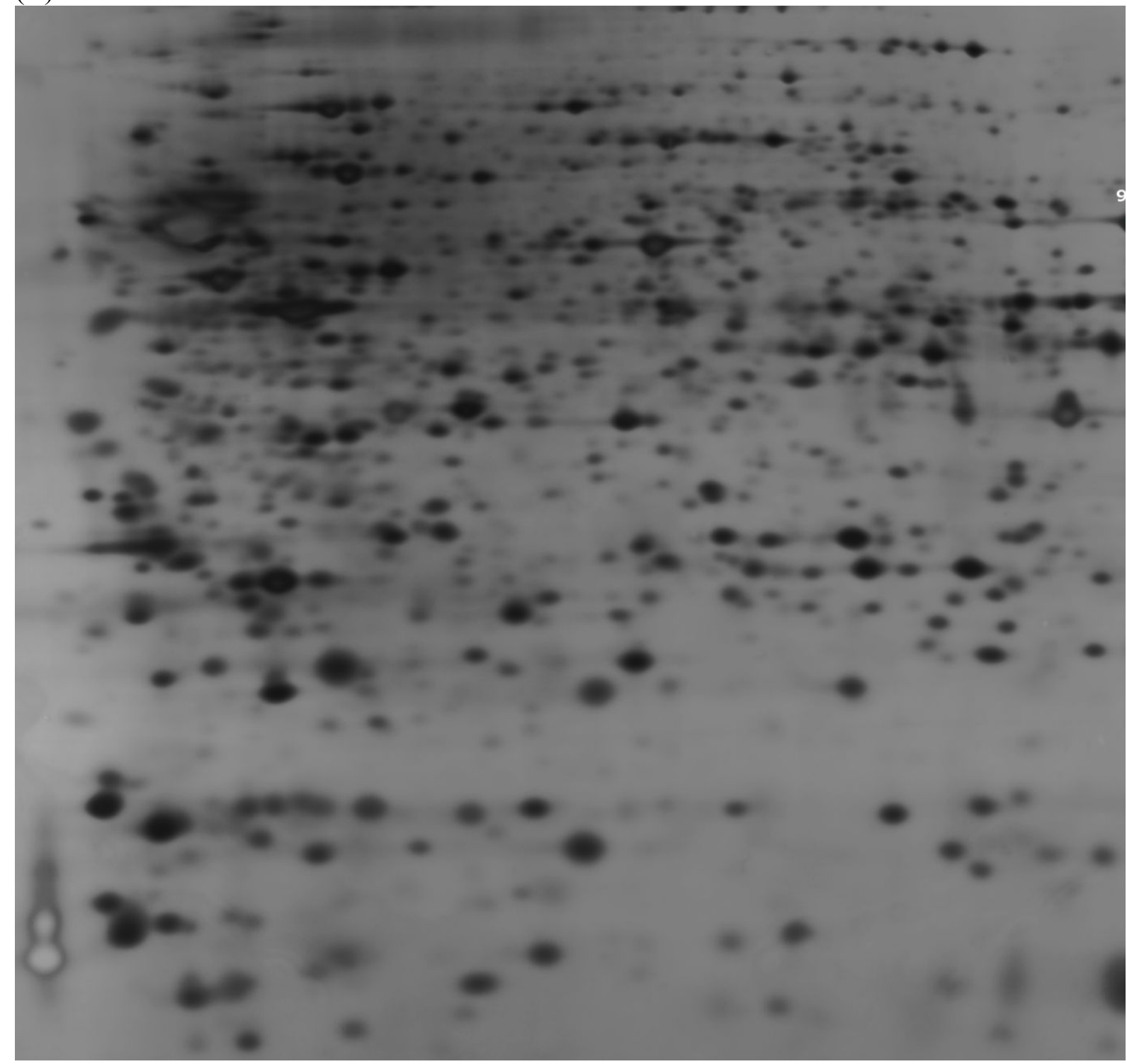

Figure S2. The right-side was compared to the left-side of CeA of (A) Naïve rats and at (B) day 7 and (C) day 14 post SNT surgery. Three replicates of silver stained 2D-gel of the right-side CeA were aligned using Progensis SameSpot software after compared to aligned 2D-gel from the left side of $\mathrm{CeA}$ and the spots with $\mathrm{F} \geq 2$ were detected by the cross-comparison analysis between 2D-gels of naïve rats (A), rats (B) at day 7 and (C) 14 post SNT surgery. The first dimension, an isoelectric focusing (IEF), was pefomed with an immobilised $\mathrm{pH}$ gel $(\mathrm{pH} 3-11 \mathrm{NL})$ of $11 \mathrm{~cm}$ long, where proteins separated according to $\mathrm{p} /$ values. The second dimension, SDS-PAGE was perfomed, with a $12 \%$ SDS polyacrylamide gel, where the proteins are separated by their molecualr weight. 


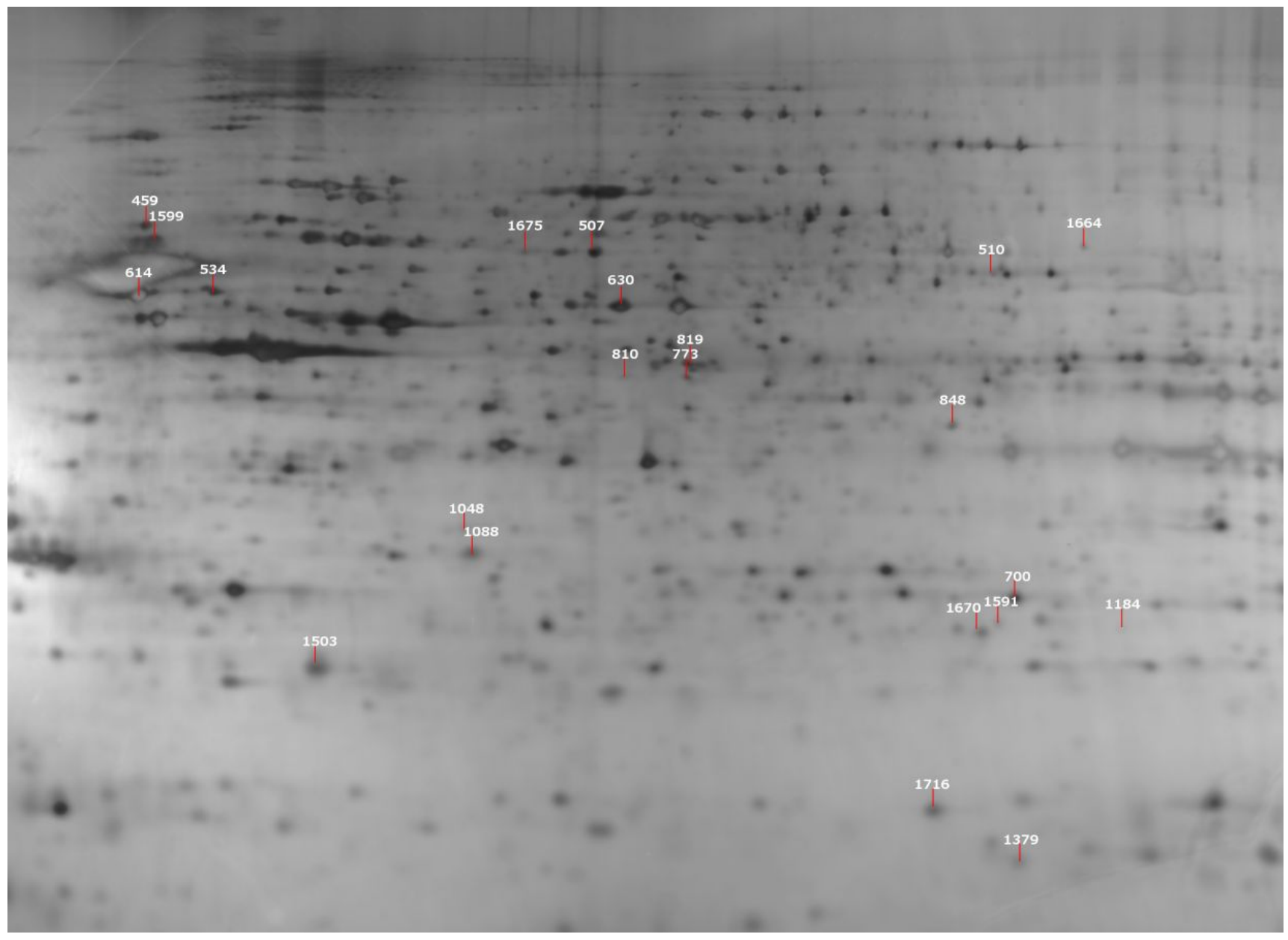

Figure S3. 2D-gel of the left-side CeA. Arrows indicate spots with fold change $\geq 2$, when 2D-gels of naïve compared to 2D-gels of neuropathic pain model rats at day 3, 7 and 21 post SNT surgery. The first dimension, an isoelectric focusing (IEF), was perfomed with a immobilised $\mathrm{pH}$ gel $(\mathrm{pH} 3-11 \mathrm{NL})$ of $24 \mathrm{~cm}$ long. The second dimension, SDS-PAGE, was perfomed with a $12 \%$ SDS polyacrylamide gel. 


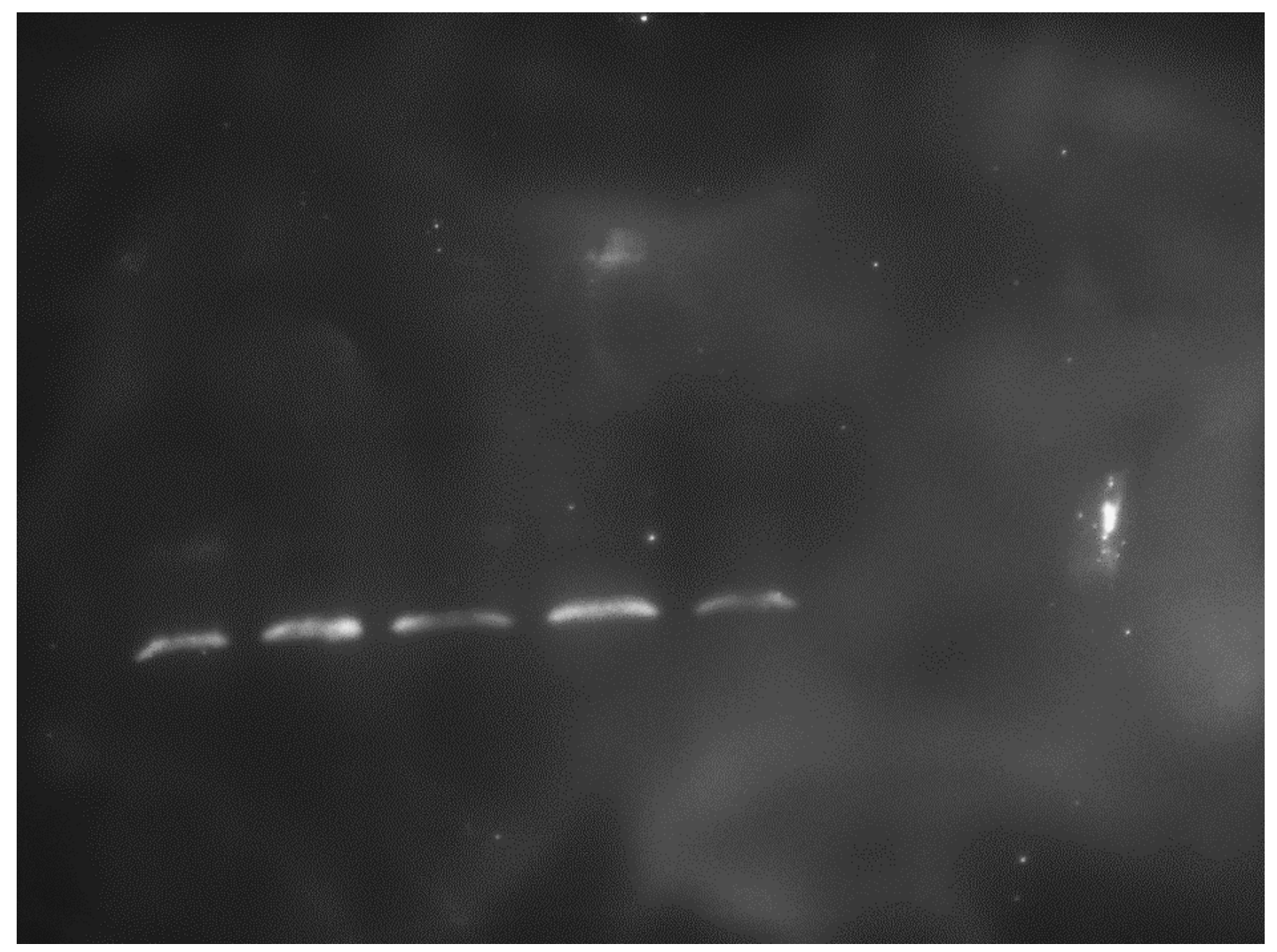

Figure S4. The entire membrane image for the Western blotting (GAPDH) shown in Figure 10. 